\title{
PLATAFORMAS DE ACCESO TRANSANAL EN CIRUGÍA COLORRECTAL: DE LA RESECCIÓN LOCAL A LA RESECCIÓN TOTAL DEL MESORRECTO*
}

\author{
Drs. José Tomás Larach K. ${ }^{1}$, Felipe Bellolio R. ${ }^{1}$ \\ 1 Departamento de Cirugía Digestiva, Unidad de Cirugía Colorrectal. Pontificia Universidad Católica de Chile. \\ Santiago, Chile.
}

\begin{abstract}
\section{Transanal access platforms in colorectal surgery: from local resection to total mesorectal excision}

Transanal access platforms have been an important contribution since their creation over 30 years ago, yielding high quality, full-thickness rectal resections in more proximal locations, with excellent visibility compared to classical transanal excision and some endoscopic techniques, in both benign and malignant lesions. Laparoscopic surgery has contributed to the emergence of new platforms that integrate conventional laparoscopic instruments into transanal resections reporting good initial results. These platforms are the present and future of natural orifice transluminal endoscopic surgery (NOTES). In this review we discuss about the different available platforms, their indications and results.

Key words: Rectal cancer, local resection, transanal resection, transanal total mesorectal excision, minimally invasive surgery.

\section{Resumen}

Las plataformas de acceso transanal han sido un aporte importante desde su creación de hace más de 30 años, permitiendo realizar resecciones transanales de pared completa de alta calidad, en localizaciones más proximales, con excelente visualización y con resultados favorables en comparación a la resección transanal clásica y algunas técnicas endoscópicas, tanto en lesiones benignas como malignas. Los avances de la cirugía laparoscópica han contribuido al desarrollo de nuevas plataformas que integran el instrumental y torres de laparoscopia convencionales, comunicando buenos resultados iniciales. Estas plataformas son el presente y futuro de la cirugía por orificios naturales o natural orifice transluminal endoscopic surgery (NOTES). En esta revisión se exponen las diferentes plataformas disponibles, sus indicaciones y resultados.

Palabras clave: Cáncer de recto, resección local, resección transanal, resección total del mesorrecto, cirugía mínimamente invasiva.

\footnotetext{
*Recibido el 28 de mayo de 2014 y aceptado para publicación el 14 de septiembre de 2014.

Los autores declaran no tener conflicto de interés, ni haber recibido aportes financieros.

Correspondencia: Felipe Bellolio R.

fbelloli@med.puc.cl
} 


\section{Introducción}

La resección total del mesorrecto (RTM) ${ }^{1}$ es el estándar del tratamiento quirúrgico del cáncer de recto medio e inferior. La RTM, como resección anterior baja o resección abdomino-perineal, abierta o laparoscópica, tiene un $28 \%$ de morbilidad, incluyendo un $7 \%$ de filtración anastomótica, $5,4 \%$ de reoperaciones y $1,8 \%$ de mortalidad $^{2}$. Se ha comunicado hasta un $51 \%$ de síndrome de resección anterior mayor en ausencia de radioterapia neoadyuvante, que se correlaciona con un deterioro significativo de la calidad de vida de estos pacientes ${ }^{3,4}$, además de porcentajes variables de disfunción urinaria y sexual producto de la disección pélvica ${ }^{5,6} \mathrm{y}$ morbilidad y rehospitalizaciones derivadas de la confección de una ileostomía de protección ${ }^{7,8}$. Por todo esto, desde hace algunas décadas, la resección local transanal (RTA) del cáncer de recto ha cautivado el interés de los cirujanos con el afán de evitar la RTM y su morbilidad asociada, sin comprometer el pronóstico oncológico en casos estrictamente seleccionados.

En 1983 se diseñó la primera plataforma de acceso transanal (PAT) para microcirugía transanal endoscópica o transanal endoscopic microsurgery (TEM), descrita por Gerhard Buess 9 . TEM fue una de las primeras formas de cirugía mínimamente invasiva, y aunque fue concebida para realizar RTA de grandes adenomas rectales, no resecables por vía endoscópica, rápidamente se empezó a utilizar en el cáncer de recto inicial (T1-2, N0) con resultados variables. A pesar de que la mayor parte de la evidencia del uso de las PAT se funda en TEM, su difusión no fue tan espectacular como se proyectó inicialmente por su alto costo y complejo aprendizaje, y su desarrollo se ha llevado a cabo principalmente en centros de alto volumen, con cirujanos especializados. Últimamente han surgido PAT más económicas, que utilizan el instrumental laparoscópico convencional, revolucionando el campo de la cirugía colorrectal con un número creciente de publicaciones que avalan su efectividad y seguridad ${ }^{10-16}$. Además, se han extendido las indicaciones a otras patologías rectales benignas y malignas, al cáncer de recto avanzado y se han usado como puente para cirugía por orificios naturales o natural orifice tranluminal endoscopic surgery (NOTES) $)^{11,13-19}$.

\section{Plataformas de acceso transanal (Tabla 1)}

\section{Transanal endoscopic microsurgery (TEM)} (Richard Wolf Medical Instruments, Chicago, Illinois, Estados Unidos)

Consta de un rectoscopio biselado, rígido, de $4 \mathrm{~cm}$ de diámetro, y de 10 ó $20 \mathrm{~cm}$ de largo, que permite acceso a lesiones ubicadas hasta $20 \mathrm{~cm}$ del margen anal. Entrega distensión rectal controlada, mediante un insuflador de $\mathrm{CO}^{2}$ y aspirador especialmente diseñado. Entrega buena visibilidad mediante una imagen 3D magnificada a través de binoculares o una imagen 2D proyectada en un monitor. Utiliza instrumental angulado especial a través de tres canales de trabajo en el rectoscopio (y un cuarto dedicado a una cámara de $30^{\circ}$ o $45^{\circ}$ ), incluyendo pinzas, tijeras, aspirador/irrigador, electrocoagulador, porta-agujas, etc. (Figura 1).

\section{Transanal endoscopic operation (TEO) (Karl Storz GmbH, Tuttlingen, Alemania)}

Introducida el 2008, permitió incorporar instrumental laparoscópico convencional en la RTA. TEO es una plataforma que recoge los principios básicos del TEM (Figura 2). Consta de un rectoscopio biselado, rígido, de 7,5, 15 ó $20 \mathrm{~cm}$ de largo y de $4 \mathrm{~cm}$ de diámetro, con tres canales de trabajo de 12,5 y $5 \mathrm{~mm}$ y otro dedicado para una cámara de $30^{\circ}$. El rectoscopio se conecta a la mesa operatoria mediante un brazo articulado. El sistema se usa en combinación con torres de laparoscopia convencionales. La imagen 2D es proyectada en una pantalla y se utiliza $\mathrm{CO}^{2}$ para la insuflación ${ }^{20}$.

\section{Transanal minimally invasive surgery (TAMIS)}

TAMIS es un híbrido entre TEM y la cirugía laparoscópica de puerto único utilizada vía transanal.

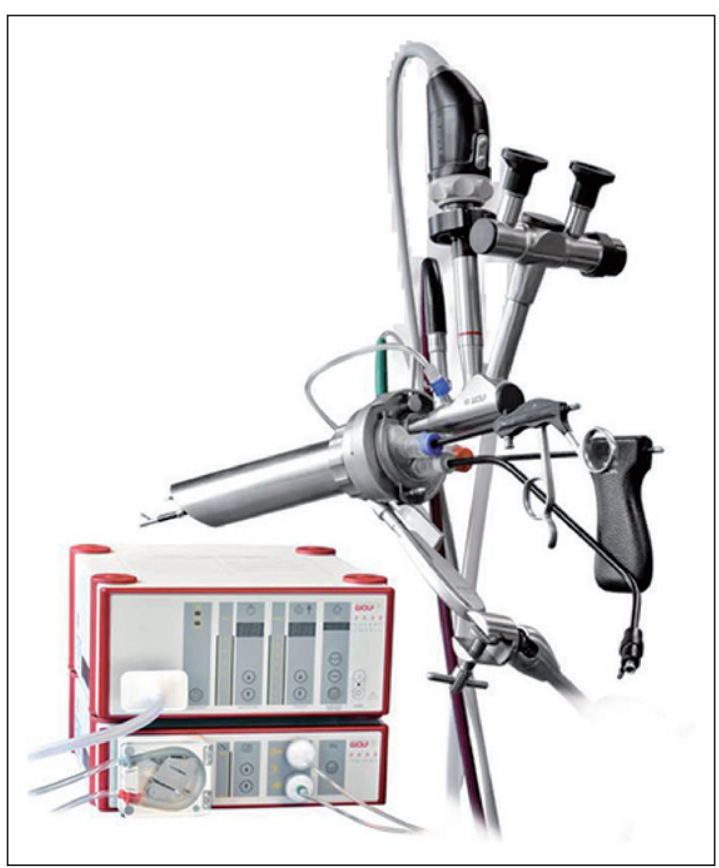

Figura 1. TEM. 


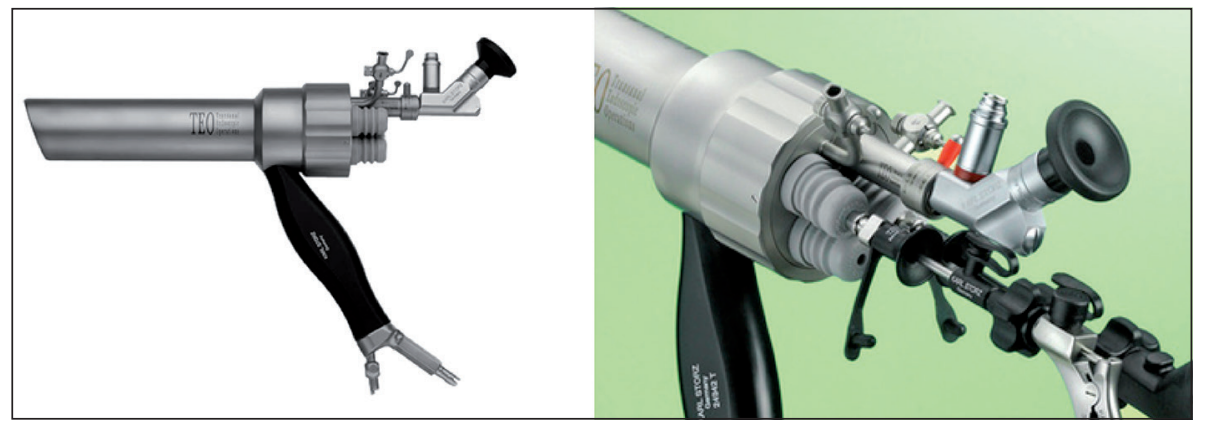

Figura 2. TEO.
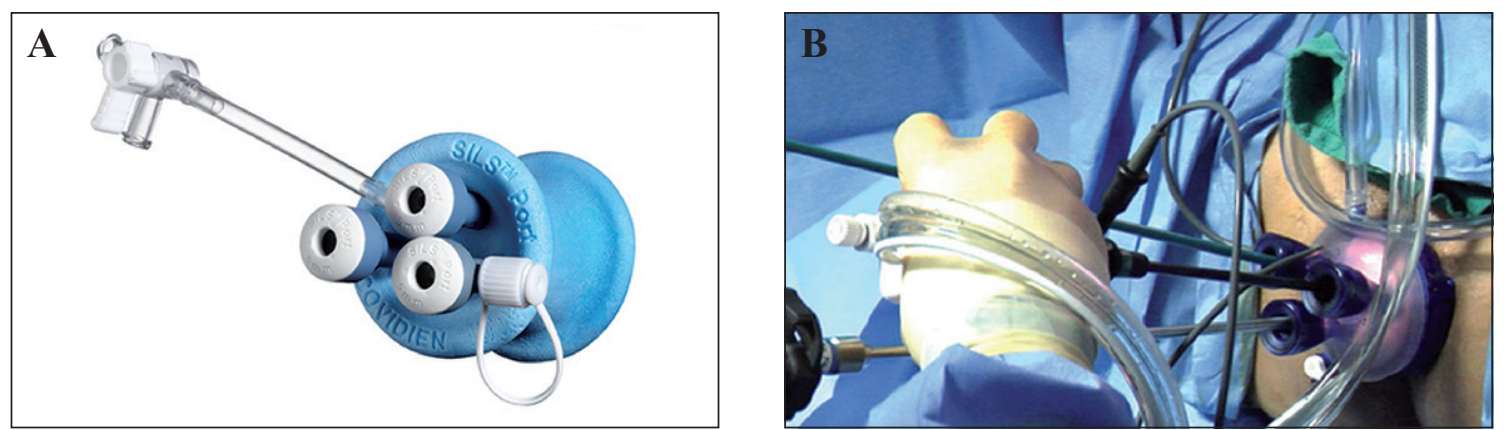

Figura 3. Dispositivos flexibles para TAMIS. A. SILS ${ }^{\text {TM }}$ Port. B. Acceso transanal a través del GelPOINT® Path.

El concepto fue desarrollado el 2009 y publicado el $2010^{12}$, facilitado por la existencia de dos dispositivos; SILS ${ }^{\mathrm{TM}}$ Port (Covidien, Mansfield, MA, Estados Unidos) desarrollado inicialmente para cirugía laparoscópica de puerto único y GelPOINT® Path (Applied Medical, Rancho Santa Margarita, CA, Estados Unidos) diseñado específicamente para TAMIS. Estos dispositivos permiten utilizar cámaras, insufladores e instrumental laparoscópico convencional (Figura 3).

GelPOINT® Path utiliza tres puertos para instrumental de 5 ó $12 \mathrm{~mm}$, que se insertan a través de una interfaz gelatinosa y cuenta con un puerto lateral para insuflar $\mathrm{CO}^{2}$ hasta $18 \mathrm{mmHg}$. Se ha descrito su uso con un endoscopio para desplazar al camarógrafo de la zona de trabajo del cirujano ${ }^{21}$. También se han comunicado métodos más económicos de TAMIS utilizando retractores de herida, anoscopios desechables y guantes quirúrgicos convencionales ${ }^{22,23}$.

\section{Indicaciones}

\section{Grandes adenomas rectales}

Las PAT han probado ser eficientes y tener baja morbilidad en el tratamiento de grandes adenomas rectales. Estudios no controlados han demostrado que TEM se asocia a menores tasas de fragmentación del espécimen, márgenes positivos y recidiva local que la RTA clásica, sin aumentar las complicaciones $^{24,25}$. La recidiva local de adenomas rectales después de una RTA clásica varía entre un $22 \%$ y $32 \%$, versus $3 \%$ y $16 \%$ para $\mathrm{TEM}^{24-27}$. El promedio de las lesiones resecadas se encuentra bajo los $5 \mathrm{~cm}$ del margen anal en la RTA clásica y sobre los $5 \mathrm{~cm}$ en $\mathrm{TEM}^{24,28}$.

Una revisión sistemática que incluyó 1.682 adenomas rectales tratados mediante TEM, comunicó una recidiva local promedio de $6,3 \%$ a 12 meses $^{27}$. Esta se asocia a resecciones incompletas, lesiones de gran tamaño y más proximales ${ }^{24,29} \mathrm{y}$ puede ser tratada mediante re-TEM de forma exitosa ${ }^{29,30}$.

Aunque no hay estudios comparativos entre resección mucosa endoscópica (RME) y RTA mediante PAT para grandes adenomas rectales, una revisión sistemática y metanálisis que incluyó 20 series de RME y 48 de TEM en lesiones rectales benignas, demostró que a pesar de las mayores tasas de complicación postoperatoria para TEM ( $13 \%$ vs $3,8 \%, \mathrm{p}<0,001)$, el tamaño promedio de los pólipos tratados fue mayor ( $37 \mathrm{~mm}$ vs $31 \mathrm{~mm}, \mathrm{p}=0,02)$ y la recidiva local a los 3 meses post-procedimiento fue menor que para RME $(5,4 \% \text { vs } 11,2 \%, \mathrm{p}=0,04)^{31}$. 
Tabla 1. Comparación entre las PAT más utilizadas

\begin{tabular}{|lll|}
\hline TEM & TEO & TAMIS \\
\hline Plataforma rígida & Plataforma rígida & Plataforma flexible \\
\hline Rectoscopio $4 \mathrm{~cm}$ diámetro & Rectoscopio $4 \mathrm{~cm}$ diámetro & Puerto $3,5-4 \mathrm{~cm}$ diámetro \\
\hline Acceso hasta $15-20 \mathrm{~cm}$ & Acceso hasta $15-20 \mathrm{~cm}$ & Acceso hasta $12 \mathrm{~cm}$ \\
\hline Visión en $220^{\circ}$ & Visión $220^{\circ}$ & Visión $360^{\circ}$ \\
\hline Imagen 2D o 3D (binocular) & Imagen 2D & Imagen 2D \\
\hline Insuflador CO ${ }^{2}$ especializado & Insuflador $\mathrm{CO}^{2}$ laparoscópico & Insuflador CO $\mathrm{CO}^{2}$ laparoscópico \\
\hline Instrumental especializado & Instrumental laparoscópico & Instrumental laparoscópico \\
\hline Posición “tumor abajo" & Posición “tumor abajo" & Litotomía \\
\hline Alto costo, reutilizable & Alto costo, reutilizable & Bajo costo, desechable \\
\hline
\end{tabular}

Actualmente está en curso un estudio multicéntrico, randomizado, que pretende comparar los resultados de ambas técnicas en adenomas rectales ${ }^{32}$.

Dado que se ha comunicado hasta un $8,4 \%$ de lesiones invasoras no detectadas antes de la resección local de adenomas rectales ${ }^{30}$ y hasta un 54\% de resección fragmentada, con mayores tasas de resecciones $\mathrm{R} 1$ y recidiva local que la resección no fragmentada en las series de $\mathrm{RME}^{33}$, la técnica endoscópica más adecuada a comparar con las PAT debiera ser la disección endoscópica submucosa (DES) y no la RME. DES permite la resección no fragmentada de lesiones, pero es técnicamente más demandante y tiene mayores complicaciones que la $\mathrm{RME}^{34}$. Una revisión sistemática y metanálisis incluyó 11 series de DES y 10 series de TEM en pacientes con adenomas rectales ${ }^{35}$. La resección no fragmentada y resección $\mathrm{R} 0$ fue significativamente mayor para TEM, sin diferencias en el número de complicaciones. La recidiva reportada es menor para la DES que para TEM $(2,6 \%$ vs $5,2 \%$, p < 0,001), pero la mediana de seguimiento para ambos grupos es distinta, de manera que esta comparación pierde valor. Los autores concluyen que a pesar de que DES es un procedimiento seguro, TEM logra mejores tasas de resección R0 cuando se realiza resección de pared total ${ }^{35}$.

El estudio preoperatorio de lesiones benignas es inexacto para descartar un componente invasor ${ }^{31}$. Cuando se ha realizado una resección parietal parcial (especialmente con técnicas endoscópicas) con histología post-procedimiento de adenocarcinoma invasor, la RTA de pared completa del lecho mediante PAT, puede determinar mejor el nivel de invasión y necesidad de una resección radical o seguimiento según los hallazgos histopatológicos ${ }^{36}$.

\section{Cáncer de recto inicial}

La RTA es una alternativa atractiva en el tratamiento del cáncer de recto, dado que tiene significativamente menos complicaciones ${ }^{37}$ y mejores resultados funcionales que la RTM.

Al igual que en lesiones benignas, la RTA mediante PAT permite mejor visualización de las lesiones, menor fragmentación de especímenes, acceso a lesiones mas proximales y menor recidiva local que la RTA clásica en lesiones malignas ${ }^{10,25,26,28,38}$.

Dos estudios retrospectivos con un número pequeño de pacientes han comparado la RTA mediante PAT y DES en lesiones malignas, concluyendo que ambos procedimientos son seguros y efectivos en el tratamiento de cáncer de recto inicial, sin lograr demostrar superioridad de uno u otro ${ }^{39,40}$.

A pesar de sus potenciales beneficios, el rol oncológico de la RTA en el cáncer de recto es controversial, ya que la resección de pared completa no contempla una linfadenectomía adecuada, aumentando el riesgo de recidiva local ${ }^{37,41-43}$. A pesar de esto, la RTA en cáncer de recto en Estados Unidos ha aumentado significativamente en los últimos años, con altas tasas de recidiva local $(12,5 \%$ para $\mathrm{T} 1 \mathrm{y}$ $22,1 \%$ para T2 a 5 años) $)^{37}$.

Factores de riesgo histológicos clásicos para la presencia de adenopatías y recidiva local en cáncer de recto inicial son la profundidad de invasión en la submucosa (sm2-3), lesión T2, infiltración linfovascular y pobre diferenciación tumoral ${ }^{44-48}$. Estos son denominados factores histológicos de alto riesgo, mientras que los tumores que no presentan estas características son de bajo riesgo ${ }^{49}$. Otros factores asociados a recidiva local son el tamaño tumoral $\geq 3$ $\mathrm{cm}$, localización en el recto distal, sexo masculino, consumo de alcohol y ulceración tumoral ${ }^{44,48,50,51}$. La 
presencia de adenopatías en tumores de bajo riesgo es de $7,5 \%$ y $15,3 \%$ para $\mathrm{T} 1$ y $\mathrm{T} 2$, respectivamente; en la medida en que se agregan factores de riesgo histológicos, la presencia de adenopatías puede sobrepasar el $60 \%$ en ambos casos, especialmente en $\mathrm{T} 22^{47}$

La recidiva local de cáncer de recto inicial postRTA cobra importancia al haber evidencia de que la RTM de salvataje tiene peor pronóstico oncológico en pacientes que de haber sido sometidos a RTM inicialmente tendrían excelente sobrevida global y libre de enfermedad. Re-TEM ante una recidiva local ha demostrado peor sobrevida a largo plazo que la RTM de salvataje ${ }^{52}$. Por otro lado, la RTM de salvataje tiene mayor riesgo de resección abdominoperineal, colostomías, recidiva a distancia y disminución de sobrevida libre de enfermedad en comparación a la RTM primaria ${ }^{52-54}$. Otras series muestran mejores resultados en la sobrevida global cuando la RTM de salvataje se acompaña de un tratamiento multimodal con radioquimioterapia neoadyuvante ${ }^{55}$.

Una revisión sistemática y metanálisis incluyó 942 pacientes de 10 estudios comparativos entre TEM y RTM para cáncer de recto inicial ${ }^{56}$. Los resultados demostraron significativamente mayores tasas de recidiva local y recidiva global en aquellos pacientes sometidos a TEM. Hubo también diferencias a favor de la RTM en sobrevida global, pero ésta no fue estadísticamente significativa. Aunque destaca la heterogeneidad de los estudios en cuanto a la selección de pacientes y el uso de radioterapia neoadyuvante, se puede concluir que la RTA no parece tener un rol en todos los pacientes con cáncer de recto inicial.

Un metanálisis de 5 estudios comparativos, incluyendo 397 pacientes, entre TEM y RTM para el tratamiento de cánceres de recto $\mathrm{T} 1$, demostró significativamente menor morbilidad para TEM que para RTM (8,2 vs 47,2\%, p =0,01), pero significativamente mayor recidiva local $(12 \%$ vs $0,5 \%, \mathrm{p}=$ $0,004)^{57}$. Las recidivas post-TEM reportadas oscilan entre $4 \%$ y $18 \%$, lo que se explica probablemente por las diferencias entre tumores $\mathrm{T} 1$ de bajo riesgo $\mathrm{y}$ alto riesgo.

La RTA mediante PAT en cáncer de recto T1 de bajo riesgo ha demostrado una tasa de recidiva local similar a la RTM (4\% vs 3\%, respectivamente) ${ }^{42}$. En un estudio, la recidiva de tumores $\mathrm{T} 1$ de bajo riesgo fue de $6 \%$, mientras que en tumores de alto riesgo (márgenes positivos o alto riesgo histológico) fue de 39\% a 10 años; cuando se indicó RTM inmediata en aquellos pacientes de alto riesgo, la recidiva local bajó a $6 \%(p=0,015)^{58}$. Así, la RTA mediante PAT en tumores $\mathrm{T} 1$ de bajo riesgo tiene resultados oncológicos similares a la RTM ${ }^{42,58}$ y la RTM indicada después de la RTA cuando hay factores histológicos de alto riesgo, no parece asociarse a un peor pronóstico oncológico que la RTM primaria ${ }^{58,59}$.

La RTA mediante PAT no es un tratamiento adecuado en pacientes con tumores $\mathrm{T} 2$, con recidivas locales de hasta $50 \%$ y significativamente menor sobrevida libre de enfermedad a 5 y 10 años que la $\mathrm{RTM}^{60}$.

A pesar de que se han comunicado buenos resultados con el uso de radioterapia perioperatoria en tumores $\mathrm{T} 1$ de alto riesgo, T2 y cáncer de recto avanzado tratados por RTA mediante PAT (especialmente en tumores ypT0-2, G1-2), esto sólo debiera considerarse en protocolos de investigación, ya que hay pocos estudios randomizados y la selección de pacientes, tratamientos y resultados son heterogéneos $^{61-66}$.

\section{Resección total del mesorrecto transanal}

La evolución de la laparoscopia y la técnica quirúrgica ha dado origen a NOTES, la extracción de especímenes por orificios naturales o natural orifice specimen extraction (NOSE) y cirugía por orificios naturales asistida por laparoscopia o minilaparoscopy-assisted natural orifice surgery (MANOS).

La fusión de los conceptos de RTM ${ }^{1}$, integración de PAT para NOTES y NOSE ${ }^{67}$ y movilización del recto distal mediante el abordaje transabdominal y transanal (TATA) $)^{68}$ "bottom-to-up" o "downto-up" 69 ha permitido el desarrollo de este nuevo enfrentamiento del cáncer de recto. Esta técnica fue probada con éxito en modelos cadavéricos ${ }^{70-72}$ y se ha popularizado como alternativa atractiva en pacientes obesos y/o de pelvis estrecha, donde la movilización del recto distal y adecuada visualización es técnicamente demandante, amenazando los planos oncológicos.

Se han comunicado casos de RTM mediante NOTES "puro", sin asistencia laparoscópica, utilizando TEO y TAMIS ${ }^{73,74}$. Por el momento, los puntos en contra de este abordaje son su gran dificultad técnica, lograr una movilización completa del ángulo esplénico del colon y la confección de una ileostomía de protección.

La mayoría de las RTM transanal reportadas han utilizado asistencia laparoscópica. Esto se ha denominado "abordaje híbrido" y los resultados a corto plazo confirman su efectividad y seguridad en grupos con experiencia en cirugía colorrectal laparoscópica avanzada ${ }^{13,19,69,75-79}$. También se ha descrito el uso de PAT combinadas con robótica para RTM en humanos ${ }^{14}$.

En general, se establece primero el margen distal y se confecciona una jareta 1 a $2 \mathrm{~cm}$ bajo el tumor. Se realiza una incisión circunferencial sobre el anillo anorrectal (o resección interesfinteriana dependiendo de la altura del tumor) para entrar al plano mesorrec- 

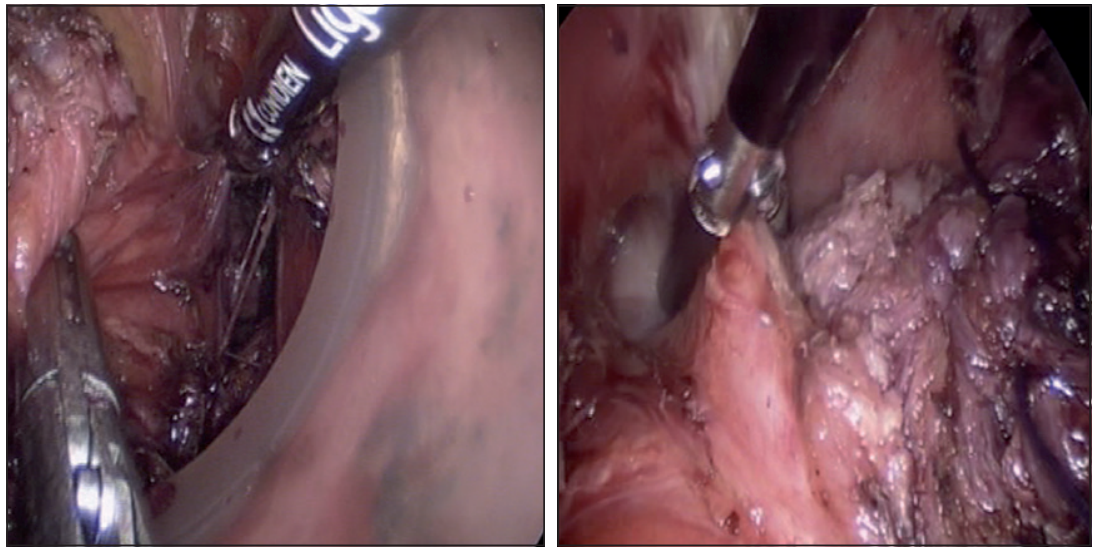

Figura 4. RTM transanal mediante TAMIS. Visión del cirujano movilizando circunferencialmente el recto inferior y medio vía transanal. La ligadura de la arteria mesentérica inferior, descenso del ángulo esplénico del colon y confección de una ileostomía de protección (si es necesaria) se realizan por laparoscopia.

tal. Se inserta la plataforma para continuar la disección vía transanal "down-to-up" ${ }^{69}$ con la insuflación de $\mathrm{CO}^{2}$ y disección cortante utilizando instrumentos bipolares o monopolares, estableciendo un plano sin violar la fascia mesorrectal (Figura 4). La disección laparoscópica y transanal pueden ser simultáneas, optimizando el tiempo quirúrgico. Una vez terminada la disección, se extrae el espécimen vía transanal y se realiza una anastomosis manual o mecánica. La técnica no está estandarizada y la práctica de cirugía laparoscópica avanzada no garantiza la adquisición inmediata de habilidades para RTM transanal.

\section{Otras}

TEM y TEO se han usado en estenosis benignas rectales (postquirúrgicas), drenaje de abscesos pélvicos, GIST y tumores neuroendocrinos rectales, proctectomías reversas en enfermedad inflamatoria intestinal, evacuación de fecalomas impactados, resolución de fístulas rectouretrales, resección de condilomas acuminados endorrectales, tumores retrorrectales o presacros, prolapso rectal y reparación de perforaciones traumáticas o iatrogénicas del recto ${ }^{80,81}$. TAMIS ha sido utilizado con éxito en proctectomías reversas en enfermedad inflamatoria intestinal $^{11,15}$, tratamiento de lesiones vasculares rectales, fístulas rectouretrales y extracción de cuerpos extraños rectales ${ }^{11}$.

\section{Complicaciones}

Las complicaciones de la RTA mediante PAT son poco frecuentes $(6,4$ a $11,8 \%)$ y menores $^{82,83}$, siendo las más habituales el sangrado intraoperatorio, especialmente asociado a resección de grandes lesiones, sangrado postoperatorio y retención urinaria ${ }^{83}$. La ubicación lateral de las lesiones se ha asociado a un aumento de complicaciones hemorrágicas ${ }^{82}$. La fístula rectovaginal es una complicación temida, comunicada en alrededor del $1 \%$ de los $\operatorname{casos}^{82,83}$. La entrada a la cavidad peritoneal ya no es considerada una complicación del procedimiento, ni tampoco un factor de deterioro oncológico ${ }^{84,85}$; por el contrario, sería un modo seguro de acceso a la cavidad peritoneal para NOTES, con bajo riesgo de complicaciones ${ }^{17}$.

Se han comunicado mayores complicaciones de la herida operatoria en pacientes sometidos a radioterapia neoadyuvante y RTA en cáncer de recto, pero la mayoría son manejadas de forma conservadora, siendo la ostomía derivativa algo inusual ${ }^{86}$.

Las complicaciones comunicadas de RTM transanal no difieren de la RTM laparoscópica convencional $^{13,19,69,75-79}$.

\section{Resultados funcionales}

Uno de los argumentos centrales para justificar la RTA mediante PAT sobre la RTM en cáncer rectal es la preservación de la función anorrectal. De todas formas, existe preocupación por el daño funcional que podrían provocar las PAT al requerir la introducción de un rectoscopio rígido o dispositivos flexibles y la insuflación constante de $\mathrm{CO}^{2}$ en el recto por tiempos prolongados.

Se han reportando alteraciones de la presión de reposo, contracción máxima y volumen máximo tolerable en la manometría anorrectal a corto plazo utilizando TEM, pero que no se correlacionan con los puntajes de incontinencia o calidad de vida, y tienden a recuperarse gradualmente desde los 3 meses post-procedimiento ${ }^{87-89}$. Los puntajes de incontinencia y calidad de vida evaluados en múltiples estudios, no demuestran alteración significativa después del uso de TEM en el corto o largo pla$\mathrm{zo}^{90,91}$. Además, TEM ha demostrado no deteriorar 
la función anorrectal después de procedimientos repetidos ${ }^{92}$.

Algunos estudios han comparado los resultados funcionales entre TEM y RTM $^{90,93,94}$. Estos han encontrado diferencias iniciales en los puntajes de defecación (que tienden a la mejoría a mediano plazo para RTM), sin diferencias significativas en la calidad de vida entre ambos grupos.

\section{Curva de aprendizaje}

La curva de aprendizaje en TEM afecta el porcentaje de conversión, tiempo operatorio y la tasa de complicaciones ${ }^{24,95-97}$. Existe una correlación directa entre la altura de la lesión y la experiencia del cirujano con la conversión ${ }^{24}$. En procedimientos resectivos la curva de aprendizaje se estabiliza después de 4 casos en general, pero cuando las lesiones se encuentran a más de $8 \mathrm{~cm}$ del margen anal, la curva se estabiliza después de los 10 casos $^{97}$. El cierre del defecto no es evaluado en estos estudios (la sutura intracorpórea mediante PAT es más difícil que en laparoscopia por la falta de triangulación del instrumental). Actualmente hay suturas y dispositivos como el V-Loc ${ }^{\mathrm{TM}}$ y EndoStitch ${ }^{\mathrm{TM}}$ que facilitan la tarea. Por el momento, no hay estudios que evalúen la curva de aprendizaje utilizando dispositivos flexibles.

\section{Conclusiones}

Las PAT permiten realizar RTA con claras ventajas respecto a la RTA clásica y algunas técnicas endoscópicas, tanto en lesiones benignas como en cáncer de recto $\mathrm{T} 1$ de bajo riesgo, con buenos resultados oncológicos, funcionales y baja morbilidad.

Estos procedimientos son complejos, tienen una curva de aprendizaje y deben ser realizados por especialistas que conocen sus indicaciones, limitaciones y que han sido entrenados en su ejecución y manejo de sus complicaciones.

El presente y futuro de las PAT es el NOTES, ya sea puro o asistido por laparoscopia (MANOS). El desarrollo de este concepto está ligado al avance de la tecnología y ya vemos como estas plataformas se han combinado con la robótica en la resolución de patología colorrectal con proyecciones interesantes $^{14,18}$.

\section{Referencias}

1. Heald RJ. A new approach to rectal cancer. British journal of hospital medicine. 1979;22:277-81.
2. Vennix S, Pelzers L, Bouvy N, Beets GL, Pierie JP, Wiggers T, et al. Laparoscopic versus open total mesorectal excision for rectal cancer. The Cochrane database of systematic reviews. 2014;4:CD005200.

3. Bregendahl S, Emmertsen KJ, Lous J, Laurberg S. Bowel dysfunction after low anterior resection with and without neoadjuvant therapy for rectal cancer: a population-based cross-sectional study. Colorectal disease: The official journal of the Association of Coloproctology of Great Britain and Ireland 2013;15:1130-9.

4. Juul T, Ahlberg M, Biondo S, Espin E, Jiménez LM, Matzel KE, et al. Low anterior resection syndrome and quality of life: an international multicenter study. Diseases of the colon and rectum 2014;57:585-91.

5. Hur H, Bae SU, Kim NK, Min BS, Baik SH, Lee KY, et al. Comparative study of voiding and male sexual function following open and laparoscopic total mesorectal excision in patients with rectal cancer. Journal of surgical oncology 2013;108:572-8.

6. Hida K, Hasegawa S, Kataoka Y, Nagayama S, Yoshimura K, Nomura A, et al. Male sexual function after laparoscopic total mesorectal excision. Colorectal disease: The official journal of the Association of Coloproctology of Great Britain and Ireland. 2013;15:244-51.

7. Paquette IM, Solan P, Rafferty JF, Ferguson MA, Davis BR. Readmission for dehydration or renal failure after ileostomy creation. Diseases of the colon and rectum. 2013;56:974-9.

8. Phatak UR, Kao LS, You YN, Rodríguez-Bigas MA, Skibber JM, Feig BW, et al. Impact of ileostomy-related complications on the multidisciplinary treatment of rectal cancer. Annals of surgical oncology 2014;21:507-12.

9. Buess G, Hutterer F, Theiss J, Bobel M, Isselhard W, Pichlmaier H. A system for a transanal endoscopic rectum operation. Der Chirurg; Zeitschrift fur alle Gebiete der operativen Medizen 1984;55:677-80.

10. Albert MR, Atallah SB, deBeche-Adams TC, Izfar S, Larach SW. Transanal minimally invasive surgery (TAMIS) for local excision of benign neoplasms and early-stage rectal cancer: efficacy and outcomes in the first 50 patients. Diseases of the colon and rectum. 2013;56:301-7.

11. Atallah S, Albert M, Debeche-Adams T, Larach S. Transanal minimally invasive surgery (TAMIS): applications beyond local excision. Techniques in coloproctology. 2013;17:239-43.

12. Atallah S, Albert M, Larach S. Transanal minimally invasive surgery: a giant leap forward. Surgical endoscopy 2010;24:2200-5.

13. Atallah S, Martin-Pérez B, Albert M, Debeche-Adams T, Nassif G, Hunter L, et al. Transanal minimally invasive surgery for total mesorectal excision (TAMIS-TME): results and experience with the first 20 patients undergoing curative-intent rectal cancer surgery at a single institution. Techniques in Coloproctology 2014;18:47380 . 
14. Atallah S, Nassif G, Polavarapu H, deBeche-Adams T, Ouyang J, Albert M, et al. Robotic-assisted transanal surgery for total mesorectal excision (RATS-TME): a description of a novel surgical approach with video demonstration. Techniques in Coloproctology. 2013;17:441-7.

15. Atallah SB, Larach S, deBeche-Adams TC, Albert MR. Transanal minimally invasive surgery (TAMIS): a technique that can be used for retrograde proctectomy. Diseases of the colon and rectum. 2013;56:931.

16. Serra-Aracil X, Mora-López L, Alcántara-Moral M, Caro-Tarrago A, Navarro-Soto S. Transanal endoscopic microsurgery with 3-D (TEM) or high-definition 2-D transanal endoscopic operation (TEO) for rectal tumors. A prospective, randomized clinical trial. International journal of colorectal disease 2014;29:605-10.

17. Fuchs KH, Meining A, von Renteln D, Fernández-Esparrach G, Breithaupt W, Zornig C, et al. Euro-NOTES Status Paper: from the concept to clinical practice. Surgical endoscopy 2013;27:1456-67.

18. Hompes R, Rauh SM, Ris F, Tuynman JB, Mortensen NJ. Robotic transanal minimally invasive surgery for local excision of rectal neoplasms. The British Journal of Surgery 2014;101:578-81.

19. Lacy AM, Adelsdorfer C, Delgado S, Sylla P, Rattner DW. Minilaparoscopy-assisted transrectal low anterior resection (LAR): a preliminary study. Surgical endoscopy 2013;27:339-46.

20. Morino M, Arezzo A, Allaix ME. Transanal endoscopic microsurgery. Techniques in coloproctology 2013;17 Suppl 1:S55-61.

21. McLemore EC, Coker A, Jacobsen G, Talamini MA, Horgan S. eTAMIS: endoscopic visualization for transanal minimally invasive surgery. Surgical endoscopy. 2013;27:1842-5.

22. Alessandro C, Daniela M, Michele M, Andrea T, Gianmarco G, Massimo S, et al. Glove port technique for transanal endoscopic microsurgery. International journal of surgical oncology. 2012;2012:383025.

23. Hompes R, Ris F, Cunningham C, Mortensen NJ, Cahill RA. Transanal glove port is a safe and cost-effective alternative for transanal endoscopic microsurgery. The British Journal of Surgery. 2012;99:1429-35.

24. de Graaf EJ, Doornebosch PG, Tetteroo GW, Geldof H, Hop WC. Transanal endoscopic microsurgery is feasible for adenomas throughout the entire rectum: a prospective study. Diseases of the colon and rectum 2009;52:1107-13.

25. Moore JS, Cataldo PA, Osler T, Hyman NH. Transanal endoscopic microsurgery is more effective than traditional transanal excision for resection of rectal masses. Diseases of the colon and rectum. 2008;51:1026-30; discussion 30-1.

26. Middleton PF, Sutherland LM, Maddern GJ. Transanal endoscopic microsurgery: a systematic review. Diseases of the colon and rectum. 2005;48:270-84.
27. Casadesus D. Surgical resection of rectal adenoma: a rapid review. World journal of gastroenterology: WJG 2009;15:3851-4.

28. Christoforidis D, Cho HM, Dixon MR, Mellgren AF, Madoff RD, Finne CO. Transanal endoscopic microsurgery versus conventional transanal excision for patients with early rectal cancer. Annals of Surgery 2009;249:776-82.

29. McCloud JM, Waymont N, Pahwa N, Varghese P, Richards C, Jameson JS, et al. Factors predicting early recurrence after transanal endoscopic microsurgery excision for rectal adenoma. Colorectal disease: The official journal of the Association of Coloproctology of Great Britain and Ireland. 2006;8:581-5.

30. Guerrieri M, Baldarelli M, de Sanctis A, Campagnacci R, Rimini M, Lezoche E. Treatment of rectal adenomas by transanal endoscopic microsurgery: 15 years' experience. Surgical endoscopy 2010;24:445-9.

31. Barendse RM, van den Broek FJ, Dekker E, Bemelman WA, de Graaf EJ, Fockens P, et al. Systematic review of endoscopic mucosal resection versus transanal endoscopic microsurgery for large rectal adenomas. Endoscopy 2011;43:941-9.

32. van den Broek FJ, de Graaf EJ, Dijkgraaf MG, Reitsma JB, Haringsma J, Timmer R, et al. Transanal endoscopic microsurgery versus endoscopic mucosal resection for large rectal adenomas (TREND-study). BMC surgery 2009;9:4.

33. Belderbos TD, Leenders M, Moons LM, Siersema PD. Local recurrence after endoscopic mucosal resection of nonpedunculated colorectal lesions: systematic review and meta-analysis. Endoscopy 2014;46:388-402.

34. Park YM, Cho E, Kang HY, Kim JM. The effectiveness and safety of endoscopic submucosal dissection compared with endoscopic mucosal resection for early gastric cancer: a systematic review and metaanalysis. Surgical endoscopy 2011;25:2666-77.

35. Arezzo A, Passera R, Saito Y, Sakamoto T, Kobayashi N, Sakamoto N, et al. Systematic review and metaanalysis of endoscopic submucosal dissection versus transanal endoscopic microsurgery for large noninvasive rectal lesions. Surgical endoscopy. 2014;28:427-38.

36. Arolfo S, Allaix ME, Migliore M, Cravero F, Arezzo A, Morino M. Transanal endoscopic microsurgery after endoscopic resection of malignant rectal polyps: a useful technique for indication to radical treatment. Surgical endoscopy. 2014;28:1136-40.

37. You YN, Baxter NN, Stewart A, Nelson H. Is the increasing rate of local excision for stage I rectal cancer in the United States justified?: a nationwide cohort study from the National Cancer Database. Annals of Surgery 2007;245:726-33.

38. Langer C, Liersch T, Suss M, Siemer A, Markus P, Ghadimi BM, et al. Surgical cure for early rectal carcinoma and large adenoma: transanal endoscopic microsurgery (using ultrasound or electrosurgery) compared to 
conventional local and radical resection. International journal of colorectal disease 2003;18:222-9.

39. Kawaguti FS, Nahas CS, Marques CF, da Costa Martins B, Retes FA, Medeiros RS, et al. Endoscopic submucosal dissection versus transanal endoscopic microsurgery for the treatment of early rectal cancer. Surgical endoscopy 2014;28:1173-9.

40. Park SU, Min YW, Shin JU, Choi JH, Kim YH, Kim JJ, et al. Endoscopic submucosal dissection or transanal endoscopic microsurgery for nonpolypoid rectal high grade dysplasia and submucosa-invading rectal cancer. Endoscopy 2012;44:1031-6.

41. Bhangu A, Brown G, Nicholls RJ, Wong J, Darzi A, Tekkis P. Survival outcome of local excision versus radical resection of colon or rectal carcinoma: a Surveillance, Epidemiology, and End Results (SEER) populationbased study. Annals of Surgery. 2013;258:563-9; discussion 9-71.

42. Heintz A, Morschel M, Junginger T. Comparison of results after transanal endoscopic microsurgery and radical resection for T1 carcinoma of the rectum. Surgical endoscopy 1998;12:1145-8.

43. Suppiah A, Maslekar S, Alabi A, Hartley JE, Monson JR. Transanal endoscopic microsurgery in early rectal cancer: time for a trial? Colorectal disease: The official journal of the Association of Coloproctology of Great Britain and Ireland. 2008;10:314-27; discussion 27-9.

44. Nascimbeni R, Burgart LJ, Nivatvongs S, Larson DR. Risk of lymph node metastasis in T1 carcinoma of the colon and rectum. Diseases of the colon and rectum. 2002;45:200-6.

45. Okabe S, Shia J, Nash G, Wong WD, Guillem JG, Weiser MR, et al. Lymph node metastasis in T1 adenocarcinoma of the colon and rectum. Journal of gastrointestinal surgery: official journal of the Society for Surgery of the Alimentary Tract. 2004;8:1032-9; discussion 9-40.

46. Sakuragi M, Togashi K, Konishi F, Koinuma K, Kawamura Y, Okada M, et al. Predictive factors for lymph node metastasis in $\mathrm{T} 1$ stage colorectal carcinomas. Diseases of the colon and rectum 2003;46:1626-32.

47. Chang HC, Huang SC, Chen JS, Tang R, Changchien $\mathrm{CR}$, Chiang JM, et al. Risk factors for lymph node metastasis in pT1 and pT2 rectal cancer: a single-institute experience in 943 patients and literature review. Annals of surgical oncology. 2012;19:2477-84.

48. Bach SP, Hill J, Monson JR, Simson JN, Lane L, Merrie A, et al. A predictive model for local recurrence after transanal endoscopic microsurgery for rectal cancer. The British Journal of Surgery 2009;96:280-90.

49. Hermanek P, Gall FP. Early (microinvasive) colorectal carcinoma. Pathology, diagnosis, surgical treatment. International journal of colorectal disease. 1986;1:79-84.

50. Doornebosch PG, Zeestraten E, de Graaf EJ, Hermsen P, Dawson I, Tollenaar RA, et al. Transanal endoscopic microsurgery for T1 rectal cancer: size matters! Surgical endoscopy 2012;26:551-7.
51. Patel SA, Chen YH, Hornick JL, Catalano P, Nowak JA, Zukerberg LR, et al. Early-stage rectal cancer: clinical and pathologic prognostic markers of time to local recurrence and overall survival after resection. Diseases of the colon and rectum. 2014;57:449-59.

52. Stipa F, Giaccaglia V, Burza A. Management and outcome of local recurrence following transanal endoscopic microsurgery for rectal cancer. Diseases of the colon and rectum 2012;55:262-9.

53. Doornebosch PG, Ferenschild FT, de Wilt JH, Dawson I, Tetteroo GW, de Graaf EJ. Treatment of recurrence after transanal endoscopic microsurgery (TEM) for T1 rectal cancer. Diseases of the colon and rectum 2010;53:1234-9.

54. Friel CM, Cromwell JW, Marra C, Madoff RD, Rothenberger DA, García-Aguilar J. Salvage radical surgery after failed local excision for early rectal cancer. Diseases of the colon and rectum 2002;45:875-9.

55. You YN, Roses RE, Chang GJ, Rodríguez-Bigas MA, Feig BW, Slack R, et al. Multimodality salvage of recurrent disease after local excision for rectal cancer. Diseases of the colon and rectum 2012;55:1213-9.

56. Sajid MS, Farag S, Leung P, Sains P, Miles WF, Baig MK. Systematic review and meta-analysis of published trials comparing the effectiveness of transanal endoscopic microsurgery and radical resection in the management of early rectal cancer. Colorectal disease: The official journal of the Association of Coloproctology of Great Britain and Ireland 2014;16:2-14.

57. Wu Y, Wu YY, Li S, Zhu BS, Zhao K, Yang XD, et al. TEM and conventional rectal surgery for T1 rectal cancer: a meta-analysis. Hepato-gastroenterology 2011;58:364-8.

58. Borschitz T, Heintz A, Junginger T. The influence of histopathologic criteria on the long-term prognosis of locally excised pT1 rectal carcinomas: results of local excision (transanal endoscopic microsurgery) and immediate reoperation. Diseases of the colon and rectum 2006;49:1492-506; discussion 500-5.

59. Gagliardi G, Newton TR, Bailey HR. Local excision of rectal cancer followed by radical surgery because of poor prognostic features does not compromise the long term oncologic outcome. Colorectal disease: The official journal of the Association of Coloproctology of Great Britain and Ireland 2013;15:e659-64.

60. Borschitz T, Heintz A, Junginger T. Transanal endoscopic microsurgical excision of pT2 rectal cancer: results and possible indications. Diseases of the colon and rectum. 2007; 50: 292-301.

61. Duek SD, Issa N, Hershko DD, Krausz MM. Outcome of transanal endoscopic microsurgery and adjuvant radiotherapy in patients with $\mathrm{T} 2$ rectal cancer. Diseases of the colon and rectum 2008;51:379-84; discussion 84 .

62. Lezoche E, Baldarelli M, Lezoche G, Paganini AM, Gesuita R, Guerrieri M. Randomized clinical trial of endoluminal locoregional resection versus laparosco- 
pic total mesorectal excision for T2 rectal cancer after neoadjuvant therapy. The British Journal of Surgery 2012;99:1211-8.

63. Ung L, Chua TC, Engel AF. A Systematic Review of local excision combined with chemoradiotherapy for early rectal cancer. Colorectal disease: The official journal of the Association of Coloproctology of Great Britain and Ireland 2014;16:502-15.

64. Borschitz T, Wachtlin D, Mohler M, Schmidberger H, Junginger T. Neoadjuvant chemoradiation and local excision for T2-3 rectal cancer. Annals of surgical oncology. 2008; 15:712-20.

65. Pucciarelli S, De Paoli A, Guerrieri M, La Torre G, Maretto I, De Marchi F, et al. Local excision after preoperative chemoradiotherapy for rectal cancer: results of a multicenter phase II clinical trial. Diseases of the colon and rectum 2013;56:1349-56.

66. Pérez RO, Habr-Gama A, Lynn PB, Sao Juliao GP, Bianchi R, Proscurshim I, et al. Transanal endoscopic microsurgery for residual rectal cancer (ypT0-2) following neoadjuvant chemoradiation therapy: another word of caution. Diseases of the colon and rectum 2013;56:6-13.

67. Han FH, Hua LX, Zhao Z, Wu JH, Zhan WH. Transanal natural orifice specimen extraction for laparoscopic anterior resection in rectal cancer. World journal of gastroenterology: WJG. 2013;19:7751-7.

68. Marks JH, Frenkel JL, D'Andrea AP, Greenleaf CE. Maximizing rectal cancer results: TEM and TATA techniques to expand sphincter preservation. Surgical oncology clinics of North America 2011;20:501-20, viii-ix.

69. de Lacy AM, Rattner DW, Adelsdorfer C, Tasende MM, Fernández M, Delgado S, et al. Transanal natural orifice transluminal endoscopic surgery (NOTES) rectal resection: "down-to-up" total mesorectal excision (TME)short-term outcomes in the first 20 cases. Surgical endoscopy 2013;27:3165-72.

70. Telem DA, Han KS, Kim MC, Ajari I, Sohn DK, Woods $\mathrm{K}$, et al. Transanal rectosigmoid resection via natural orifice translumenal endoscopic surgery (NOTES) with total mesorectal excision in a large human cadaver series. Surgical endoscopy. 2013;27:74-80.

71. Fajardo AD, Hunt SR, Fleshman JW, Mutch MG. Video. Transanal single-port low anterior resection in a cadaver model. Surgical endoscopy 2010;24:1765.

72. Bhattacharjee HK, Kirschniak A, Storz P, Wilhelm P, Kunert W. Transanal endoscopic microsurgery-based transanal access for colorectal surgery: experience on human cadavers. Journal of laparoendoscopic \& advanced surgical techniques Part A. 2011;21:835-40.

73. Leroy J, Barry BD, Melani A, Mutter D, Marescaux J. No-scar transanal total mesorectal excision: the last step to pure NOTES for colorectal surgery. JAMA surgery. 2013;148:226-30; discussion 31.

74. Zhang H, Zhang YS, Jin XW, Li MZ, Fan JS, Yang ZH.
Transanal single-port laparoscopic total mesorectal excision in the treatment of rectal cancer. Techniques in coloproctology 2013;17:117-23.

75. Tuech JJ, Bridoux V, Kianifard B, Schwarz L, Tsilividis B, Huet E, et al. Natural orifice total mesorectal excision using transanal port and laparoscopic assistance. European journal of surgical oncology: the journal of the European Society of Surgical Oncology and the British Association of Surgical Oncology. 2011;37:334-5.

76. Sylla P, Bordeianou LG, Berger D, Han KS, Lauwers GY, Sahani DV, et al. A pilot study of natural orifice transanal endoscopic total mesorectal excision with laparoscopic assistance for rectal cancer. Surgical endoscopy 2013;27:3396-405.

77. Velthuis S, van den Boezem PB, van der Peet DL, Cuesta MA, Sietses C. Feasibility study of transanal total mesorectal excision. The British Journal of Surgery. 2013;100:828-31; discussion 31.

78. Dumont F, Goere D, Honore C, Elias D. Transanal endoscopic total mesorectal excision combined with single-port laparoscopy. Diseases of the colon and rectum 2012;55:996-1001.

79. Rouanet P, Mourregot A, Azar CC, Carrere S, Gutowski $\mathrm{M}$, Quenet F, et al. Transanal endoscopic proctectomy: an innovative procedure for difficult resection of rectal tumors in men with narrow pelvis. Diseases of the colon and rectum. 2013;56:408-15.

80. Serra-Aracil X, Mora-López L, Alcántara-Moral M, Corredera-Cantarin C, Gómez-Díaz C, Navarro-Soto S. Atypical indications for transanal endoscopic microsurgery to avoid major surgery. Techniques in coloproctology 2014;18:157-64.

81. Liyanage C, Ramwell A, Harris GJ, Levy BF, Simson JN. Transanal endoscopic microsurgery: a new technique for completion proctectomy. Colorectal disease: The official journal of the Association of Coloproctology of Great Britain and Ireland. 2013;15:e542-7.

82. Kreissler-Haag D, Schuld J, Lindemann W, Konig J, Hildebrandt U, Schilling M. Complications after transanal endoscopic microsurgical resection correlate with location of rectal neoplasms. Surgical endoscopy 2008;22:612-6.

83. Kumar AS, Coralic J, Kelleher DC, Sidani S, Kolli K, Smith LE. Complications of transanal endoscopic microsurgery are rare and minor: a single institution's analysis and comparison to existing data. Diseases of the colon and rectum 2013;56:295-300.

84. Eyvazzadeh DJ, Lee JT, Madoff RD, Mellgren AF, Finne CO. Outcomes after transanal endoscopic microsurgery with intraperitoneal anastomosis. Diseases of the colon and rectum. 2014;57:438-41.

85. Morino M, Allaix ME, Famiglietti F, Caldart M, Arezzo A. Does peritoneal perforation affect short- and longterm outcomes after transanal endoscopic microsurgery? Surgical endoscopy 2013;27:181-8.

86. Marks JH, Valsdottir EB, DeNittis A, Yarandi SS, New- 
man DA, Nweze I, et al. Transanal endoscopic microsurgery for the treatment of rectal cancer: comparison of wound complication rates with and without neoadjuvant radiation therapy. Surgical endoscopy 2009;23:1081-7.

87. Jin Z, Yin L, Xue L, Lin M, Zheng Q. Anorectal functional results after transanal endoscopic microsurgery in benign and early malignant tumors. World Journal of Surgery 2010;34:1128-32.

88. Kennedy ML, Lubowski DZ, King DW. Transanal endoscopic microsurgery excision: is anorectal function compromised? Diseases of the colon and rectum 2002;45:601-4.

89. Kreis ME, Jehle EC, Haug V, Manncke K, Buess GF, Becker HD, et al. Functional results after transanal endoscopic microsurgery. Diseases of the colon and rectum 1996;39:1116-21.

90. Allaix ME, Rebecchi F, Giaccone C, Mistrangelo M, Morino M. Long-term functional results and quality of life after transanal endoscopic microsurgery. The British Journal of Surgery 2011;98:1635-43.

91. Planting A, Phang PT, Raval MJ, Brown CJ. Transanal endoscopic microsurgery: impact on fecal incontinence and quality of life. Canadian journal of surgery Journal canadien de chirurgie. 2013;56:243-8.

92. Zhang HW, Han XD, Wang Y, Zhang P, Jin ZM. Anorectal functional outcome after repeated transanal endos- copic microsurgery. World journal of gastroenterology: WJG 2012;18:5807-11.

93. Lezoche E, Paganini AM, Fabiani B, Balla A, Vestri A, Pescatori L, et al. Quality-of-life impairment after endoluminal locoregional resection and laparoscopic total mesorectal excision. Surgical endoscopy 2014;28:22734.

94. Doornebosch PG, Tollenaar RA, Gosselink MP, Stassen LP, Dijkhuis CM, Schouten WR, et al. Quality of life after transanal endoscopic microsurgery and total mesorectal excision in early rectal cancer. Colorectal disease : The official journal of the Association of Coloproctology of Great Britain and Ireland. 2007;9:5538.

95. Barendse RM, Dijkgraaf MG, Rolf UR, Bijnen AB, Consten EC, Hoff C, et al. Colorectal surgeons' learning curve of transanal endoscopic microsurgery. Surgical endoscopy 2013;27:3591-602.

96. Koebrugge B, Bosscha K, Ernst MF. Transanal endoscopic microsurgery for local excision of rectal lesions: is there a learning curve? Digestive surgery 2009;26:3727.

97. Maya A, Vorenberg A, Oviedo M, da Silva G, Wexner $\mathrm{SD}$, Sands D. Learning curve for transanal endoscopic microsurgery: a single-center experience. Surgical endoscopy 2014;28:1407-12. 